\title{
Stimulus Dependence of Disparity Coding in Primate Visual Area V4
}

\author{
Jay Hegdé and David C. Van Essen \\ Department of Anatomy and Neurobiology, Washington University School of Medicine, St. Louis, Missouri
}

Submitted 12 January 2004; accepted in final form 19 August 2004

Hegdé, Jay and David C. Van Essen. Stimulus dependence of disparity coding in primate visual area V4. J Neurophysiol 93: 620-626, 2005. First published September 1, 2004; doi:10.1152/ jn.00039.2004. Disparity tuning in visual cortex has been shown using a variety of stimulus types that contain stereoscopic depth cues. It is not known whether different stimuli yield similar disparity tuning curves. We studied whether cells in visual area V4 of the macaque show similar disparity tuning profiles when the same set of disparity values were tested using bars or dynamic random dot stereograms, which are among the most commonly used stimuli for this purpose. In a majority of V4 cells $(61 \%)$, the shape of the disparity tuning profile differed significantly for the two stimulus types. The two sets of stimuli yielded statistically indistinguishable disparity tuning profiles for only a small minority $(6 \%)$ of V4 cells. These results indicate that disparity tuning in V4 is stimulus-dependent. Given the fact that bar stimuli contain two-dimensional (2-D) shape cues, and the random dot stereograms do not, our results also indicate that V4 cells represent 2-D shape and binocular disparity in an interdependent fashion, revealing an unexpected complexity in the analysis of depth and three-dimensional shape.

\section{N T R O D U C T I O N}

Stereoscopic depth perception makes use of small disparities in the retinal location of local features in binocularly viewed images. The neurophysiological basis for stereopsis is presumed to arise from disparity-tuned neurons, which are encountered in V1 and many extrastriate visual areas (Cumming and DeAngelis 2000; Gonzalez and Perez 1998; Parker and Cumming 2001; Poggio 1995). Disparity tuning has been studied using a variety of stimulus types, most commonly with bars (with disparity cues restricted to the perimeter of the stimulus) or random dot stimuli (with disparity cues distributed across the entire random dot pattern). A diversity of tuning profiles, including near, far, tuned excitatory, and tuned inhibitory, have been reported using both stimulus types. If a given cell responds to both bars and random dot patterns, it is natural to anticipate that the shapes of the corresponding tuning profiles would be similar. Some previous studies have tested this issue on small numbers of cells and have reported tuning profiles to be qualitatively similar for the various types of stimuli used (Gonzalez and Perez 1998; Poggio 1990, 1995; Poggio et al. 1985; see DISCUSSION). However, this issue has not been systematically examined to our knowledge.

In this study, we compared the disparity tuning of neurons in area V4 of alert macaque monkeys using two types of frontoparallel stimuli: bars defined by luminance and disks defined by random dot stereograms, with both stimulus sets covering the same set of disparity values. We found that, for many V4

Address for reprint requests and other correspondence: D. Van Essen, Dept. of Anatomy and Neurobiology, Box 8108, Washington University School of Medicine, St. Louis, MO 63110 (E-mail: vanessen@brainvis.wustl.edu). neurons, the shapes of the disparity tuning profiles differ markedly and in unpredictable ways. Our testing paradigm was not designed to explore the neural mechanism underlying these differences or their functional significance. Nonetheless, the findings are relevant to general issues regarding the complexity of neural coding within sensory systems.

The terms "cue dependence" and "cue independence" (or form cue invariance) have been used (Albright 1992; Olavarria et al. 1992; Stoner and Albright 1992, 1993; also see Dobkins and Albright 1993) to indicate whether or not a cell's selectivity for a given attribute or parameter (e.g., direction of motion) is independent of the cue used to define that parameter (e.g., motion of a luminance-defined bar vs. a texture-defined bar). Here, we use similar terminology in discussing the tuning of V4 neurons to stereoscopic stimuli. Cue independence is computationally attractive as a strategy that allows efficient encoding and decoding of multiple stimulus attributes. However, the consistency with which this strategy is adopted in sensory systems is an empirical matter. Cue-independent tuning for direction is common in middle temporal area (MT) neurons tested with moving texture versus bars (Albright 1992; Olavarria et al. 1992; Stoner and Albright 1992, 1993; also see Dobkins and Albright 1993); however, some cells are more broadly tuned for the texture-defined motion than for luminance-defined motion (Olavarria et al. 1992). Many neurons in inferotemporal cortex show similar selectivity for shapes regardless of whether the shape cue is luminance, texture, motion, or disparity (Sáry et al. 1993; Tanaka et al. 2001). This study provides a test of cue independence in the disparity domain using stimuli in which binocular disparity is cued by edges (step changes in mean luminance) or by patterns of random dots.

\section{METHODS}

The responses of single V4 units to stereoscopic stimuli were recorded in awake, fixating monkeys. The details of the surgical and the experimental procedures have been described previously (Hegdé and Van Essen 2000, 2003). Briefly, two adult male macaques (Macaca mulatta) were used in this study. Each animal was implanted with a scleral search coil and an acrylic cranial patch using sterile surgical procedures. After the animal was fully trained in the fixation task, a small craniotomy ( $5 \mathrm{~mm}$ diam) was made over the recording site, and a recording chamber was mounted over the craniotomy. Neurophysiological recording was carried out using epoxy-coated tungsten electrodes (A-M Systems, Carlsborg, WA) with initial impedances of 3-5 MOhm (at $1 \mathrm{kHz}$ ) inserted transdurally into the cortex. All animal-related procedures used in this study were reviewed and approved in advance by the Washington University Animal Studies Committee.

\footnotetext{
The costs of publication of this article were defrayed in part by the payment of page charges. The article must therefore be hereby marked "advertisement" in accordance with 18 U.S.C. Section 1734 solely to indicate this fact.
} 


\section{Visual stimulation and recording}

Stimuli were presented dichoptically on a Sony GDM-17E11 17-in $(1,280 \times 1,024$ pixels) noninterlaced CRT display (refresh rate, 72 $\mathrm{Hz}$ ) fitted with $\mathrm{NuVision}$ (Beaverton, OR) 17SX polarized liquid crystal shutters and viewed through passive polarized eyeglasses from a distance of $58 \mathrm{~cm}$.

Units were identified as belonging to area V4 using their receptive field properties and topographical criteria (Gattas et al. 1988; Van Essen and Zeki 1978). Single V4 units were isolated based on both the shape and the amplitude of the waveform using a dual window discriminator (Bak Electronics, Germantown, MD). We recorded from every V4 unit we were able to isolate; no additional screening criteria were imposed for the initial analysis. Following the isolation of a unit, its classical receptive field (CRF) was plotted, and the cell's stimulus preferences were determined, using mouse-driven bar, grating, and/or dynamic random dot stimuli (dRDS) on the computer's monitor. For all the cells in the second monkey, the CRF was also plotted using custom-written CRF mapping software, which used a small dRDS patch as the mapping stimulus. In general, CRFs as determined by the two techniques largely agreed with each other. When they did not, usually because the given cell was unresponsive to the dRDS patches used in the automated mapping, we adopted the CRFs as determined by the manual mapping, briefly remapping the CRF manually to ensure reliability.

The stimulus set included 21 bars and 21 dRDS disk stimuli, both ranging in disparity from -1.0 (crossed, or near) to $+1.0^{\circ}$ (uncrossed, or far) in $0.1^{\circ}$ increments. An additional five control stimuli were used, consisting of a binocularly uncorrelated dRDS, a bar, and an RDS presented in either eye alone. All cells were tested using the same set of 47 stimuli, customized for the cell under study as follows. The bar stimuli had the same length, width, color, and orientation as the cell's preferred bar. For the dRDS stimuli, the color of the central disk and the color and size of the annulus were customized so as to best drive the cell. The radial size of the annulus ranged from 2 to $5^{\circ}$. The colors of the central disk and the annulus differed from each other for 78 of 128 cells $(61 \%)$ recorded from the two animals. The preferred dRDS center color differed from the cell's preferred bar color for only 3 of the 128 cells. The stimulus color/s were chosen from a palette of eight colors (with luminances measured through active liquid crystal shutters at the center of the screen using Tektronix $\mathrm{J} 17$ photometer): white $\left(6.86 \mathrm{~cd} / \mathrm{m}^{2}\right)$, red $\left(1.36 \mathrm{~cd} / \mathrm{m}^{2}\right)$, green $(4.93$ $\left.\mathrm{cd} / \mathrm{m}^{2}\right)$, blue $\left(0.63 \mathrm{~cd} / \mathrm{m}^{2}\right)$, cyan $\left(5.72 \mathrm{~cd} / \mathrm{m}^{2}\right)$, magenta $\left(1.98 \mathrm{~cd} / \mathrm{m}^{2}\right)$, yellow $\left(6.42 \mathrm{~cd} / \mathrm{m}^{2}\right)$, and black $\left(0 \mathrm{~cd} / \mathrm{m}^{2}\right)$. All stimuli were presented against a neutral gray background $\left(1.40 \mathrm{~cd} / \mathrm{m}^{2}\right)$. The cross-talk between the monocular images was low for all colors (white, $2.1 \%$; red, $2.9 \%$; green, $1.8 \%$; blue, $1.7 \%$; cyan, $1.8 \%$; magenta, $2.4 \%$; yellow, $2.4 \%$ ) and was comparable for dRDS and bars.

For all stimuli, the annulus had a fixed disparity of $0^{\circ}$, the same as the fixation spot, while the disparity of the center varied systematically from one stimulus to the next. The dots in the center and surround were identical in all other respects (except dot color, as noted above). The size and the density of the dots varied depending on size of the receptive field so as to provide the percept of a smooth surface (dot size range, $0.10-0.21^{\circ}$; dot density range, $40-60 \%$ ). The dRDS was rendered dynamically using color-lookup table animation at the refresh rate of the monitor $(72 \mathrm{~Hz})$, so that in any given frame, a random one-third of dots were invisible (i.e., rendered in the background color in both eyes), and the remaining dots were rendered in the appropriate stimulus color. No coherent motion was apparent from one frame to the next.

Fixation and vergence were monitored in each eye using a dual scleral search coil setup (Remmel Labs, Ashland, MA). The stimuli were presented in a sequential, randomly interleaved fashion for $300-400 \mathrm{~ms}$, each with a variable $300-$ to $400-\mathrm{ms}$ interstimulus interval, while the animal fixated within a window of $0.5^{\circ}$ radius for a liquid reward. Up to six stimuli per trial were presented in this fashion. To minimize the contributions of receptive field nonuniformities, if any, the spatial placement of the stimuli was systematically jittered for both bars and dRDS, so that a given presentation of each stimulus was centered on one of the four points located $25 \%$ of the CRF radius away symmetrically around the CRF center. CRF radii ranged from 0.45 to $9.65^{\circ}$ (mean, $\left.2.95^{\circ} ; n=128\right)$. CRF eccentricities ranged from 1.4 to $23.9^{\circ}$ (mean, $6.6^{\circ} ; n=128$ ).

\section{Data analysis}

Spike data were analyzed using the S-Plus (Insightful, Seattle, WA) and Matlab (The Mathworks, Natick, MA) utilities or custom-written $\mathrm{C}$ language software. Only data from the trials throughout which the animal maintained fixation were used in this study. For each cell, the evoked responses were calculated using a time window during which the overall firing rate of the given cell (across all repetitions of all stimuli) remained above background levels. Depending on the cell, this window spanned a $60-$ to $300-\mathrm{ms}$ period following the stimulus onset. The background responses were calculated from a $100-\mathrm{ms}$ time window immediately preceding the stimulus onset for all cells. The response to a given stimulus was calculated as the average net firing rate across 16 repetitions ( $<16$ but $\geq 9$ repetitions for 14 cells). A given cell was included in the analysis only if the evoked responses of the cell significantly exceeded the background responses for at least one stimulus (1-tailed $t$-test, $P<0.05$ after Bonferroni correction). Of the 128 cells recorded from the two animals, 119 cells (53 cells from the 1 st animal, and 66 cells from the 2 nd animal) passed this test and were included in this study.

\section{Indices}

The cue sensitivity index (CSI) measured the difference between the disparity tuning curves obtained using bars versus dRDS. To calculate CSI, we performed a two-way ANOVA for each cell, with disparity and stimulus type as the two factors. The $F$ ratio for the interaction factor is a parametric measure of the degree to which the two disparity tuning curves differ as a function of disparity. This $F$ ratio was used because it measures the extent to which the two tuning profiles have different shapes and is insensitive to differences in the absolute firing rates. To calculate the CSI value for a given cell, we first calculated the $F$ ratio for the interaction factor from the actual data. We then randomized the data by reassigning the spike counts from individual presentations randomly across different disparities within the same stimulus type, and recalculated the $F$ ratio (for overviews of randomization, see Edgington 1995; Manly 1991). The CSI value was defined as the actual $F$ ratio divided by the average $F$ ratio from $10^{6}$ rounds of randomization. This scaling by the average randomized $F$ ratio effectively corrected for deviations of the data set from normality. The disparity-stimulus type interaction was considered statistically significant at the level of $P<0.05$ if the randomized $F$ ratio exceeded the actual $F$ ratio in $<5 \%$ of the rounds. The statistical significance of the disparity factor was also similarly determined.

The interaction factor $F$ ratio (and by extension, the CSI) cannot distinguish disparity-dependent differences in the tuning profile from the effects of multiplicative scaling, in which the two tuning curves are scalar multiples of each other (see Dean 1981; McAdams and Maunsell 1999). We used correlation coefficient $r$ to measure the scaling between the two tuning profiles for each cell and used randomization procedures to assess the statistical significance of the correlation. Assessing multiplicative scaling by comparing scaling factors of fitted curves (cf. McAdams and Maunsell 1999) was not feasible for most V4 cells (unpublished results). Passing the multiplicative scaling test required that the two tuning curves were correlated at $P<0.05$. Note that previous studies of cue invariance have not considered multiplicative scaling as criterion for assessing cue invariance (see Albright 1992; Olavarria et al. 1992; Sáry et al. 1993). 
A cell was classified as cue-dependent if the $P$ values were $<0.05$ for both the disparity factor and the interaction factor and if it failed the multiplicative scaling test. A cell was classified as cue-independent if it had $P<0.05$ for the disparity factor and if it either had $P>$ 0.05 for the interaction factor and/or if it passed the multiplicative scaling test. All other cells were classified as not significantly tuned for disparity.

\section{Regression}

We carried out regression analysis using either conventional least squares method (S-Plus function lsfit), which took into account all data points including outliers, or using the robust regression method of least trimmed squares method (S-Plus function ltsreg; Rousseeuw 1984), which minimizes the influence of outliers.

\section{Vergence eye movements}

Vergence eye movements can potentially confound the neuronal responses to stereoscopic stimuli (Cumming and Parker 1997; Masson et al. 1997). However, vergence eye movements (calculated from the same time window as the evoked response) were not a major confound in our dataset, as assessed by four different criteria. First, the average SD of the vergence angle was small ( 0.051 for the horizontal position and 0.056 for the vertical position), and did not vary between bars and dRDS or between the two animals (MANOVA, horizontal angles $\times$ vertical angles $\times$ stimulus types $\times$ animals, $P>0.05$ for all factors). Second, vergence angles did not systematically vary with the disparity of the stimuli (correlation coefficient $r=-0.0002$ for bars; $r=0.0018$ for dRDS; $P>0.05$ in both cases). Third, the vergence angles were not correlated with the neuronal responses for the corresponding stimuli $(r=0.0002$ for bars; $r=-0.0072$ for dRDS; $P>$ 0.05 in both cases). Finally, when tested using a two-way ANOVA (disparity $\times$ stimulus type) for individual cells, vergence angles varied as a function of disparity and stimulus type (i.e., $P<0.05$ for the disparity and stimulus factors) for seven and four cells, respectively. The proportions of these cells were indistinguishable from that those expected random (binomial proportions tests, $P>0.05$ in both cases). Vergence data calculated using the time windows described above were indistinguishable from those calculated from the entire 300-ms stimulus duration ( $t$-test, $P>0.05$, data not shown).

\section{RES U L T S}

Disparity tuning of individual V4 cells for bar versus dRDS stimuli

Using stimuli of the type shown in Fig. 1, V4 cells were generally responsive to bar stimuli, dRDS disks, or both. Figure 2 shows the disparity tuning curves for the two types of stimuli for six individual V4 cells, each of which responded both to bars and to dRDS disks significantly above background levels (1-tailed $t$-test, $P<0.05$ in all cases). For each of the six cells, the disparity tuning was statistically significant for both stimulus types (1-way ANOVAs, $P<0.05$ in all cases). The cell shown in Fig. $2 A$ was broadly tuned for near disparities for both bars (solid line) and dRDS disks (dotted line). The two tuning curves were statistically indistinguishable from one another (2-way ANOVA, disparity $\times$ stimulus type; $P>0.05$ for the interaction factor). However, for many other V4 cells, the disparity tuning differed markedly for the bars versus dRDS disks, as shown by cells in Fig. $2, B-F$. For the cell shown in Fig. $2 B$, the disparity tuning profile for dRDS was a broad tuned inhibitory pattern, whereas the disparity tuning profile for the bar stimuli was mainly tuned excitatory, with a

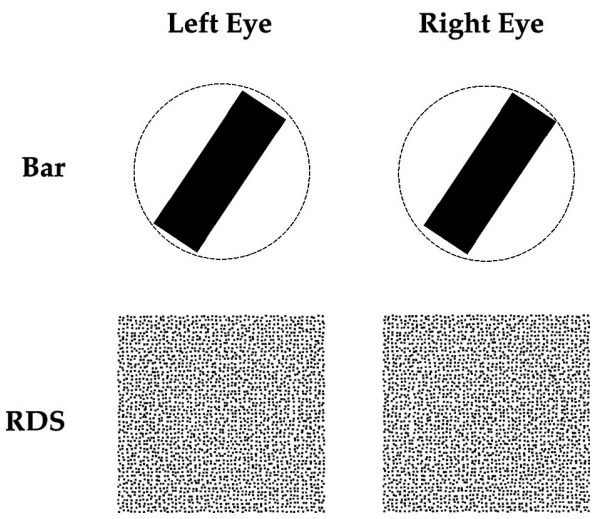

FIG. 1. Bar and random dot stereogram (RDS) stimuli. Left and the right eye views of either type of stimulus are schematically shown. Actual stimuli used in the experiments differed from the ones shown in many respects (including the fact that the dots in the RDS were dynamic); see METHODS for details. The perimeter of the classical receptive field (CRF), as determined during the manual mapping, is denoted by the dashed circle around the bar stimulus. In case of the RDS, the central disk was located within the CRF (data not shown), while the annulus was presented in the nonclassical surround. The disparity of the bars and central disk of the RDS varied systematically from one stimulus to the next, whereas the RDS annulus was always presented at zero disparity. Note that in case of bars, the disparity information is obtained by matching 2 monocular images of the bar. RDS stimuli contain no such monocularly visible shapes; in this case, disparity is cued by the optimal correspondence between the dots in the 2 monocular images.

peak near zero disparity plus smaller peaks at extreme far $\left(+1.0^{\circ}\right)$ and near $\left(-1.0^{\circ}\right)$ disparities. The difference between the two tuning profiles was highly significant (2-way ANOVA, $P<0.0001$ for interaction factor). The cells in Fig. 2, $C-F$, like that in Fig. $2 B$, also showed highly significant disparity tuning for both stimulus types, but marked differences in the shape of the tuning curves. The cell in Fig. $2 C$ showed a tuned inhibitory pattern for bars, but a tuned excitatory pattern for dRDS disks. The cell in Fig. $2 D$ had a tuned inhibitory pattern for bars and a complex (but relatively flat) response profile for dRDS disks. Cells in Fig. 2, $E$ and $F$, showed a tuned near pattern for both types of stimuli. However, the tuning profiles had different shapes and peaked at different disparities for the cell in Fig. $2 E$, whereas for the cell in Fig. $2 F$, the two tuning curves were largely scalar multiples of each other.

\section{Measuring cue sensitivity}

To assess the sensitivity of individual V4 cells to disparity cues, we measured the extent to which the disparity tuning curves of bars versus dRDS differed from one another using the cue sensitivity index (CSI; see METHODS). The CSI measured only the disparity-dependent variations between the shapes of the two tuning profiles and was, by design, insensitive to the differences in the cell's responsiveness to bars versus dRDS disks. A histogram of the CSI values for $119 \mathrm{~V} 4$ cells (Fig. 3) shows an average CSI value of 2.8 (arrow), indicating that the disparity tuning curves for the two types of stimuli differed from each other 2.8-fold more than expected from chance-level fluctuations. To assess the statistical significance of cue dependence, we tested each cell using a modified two-way ANOVA, with disparity and stimulus type as the two factors, and a multiplicative scaling test, which tested whether the two tuning curves for a given cell were scalar multiples of each other (see METHODS and Fig. 3, top). For 72 of the 119 V4 
A

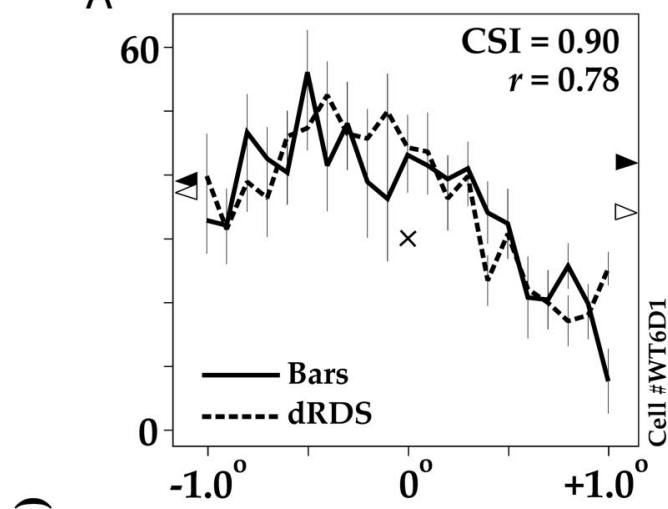

B

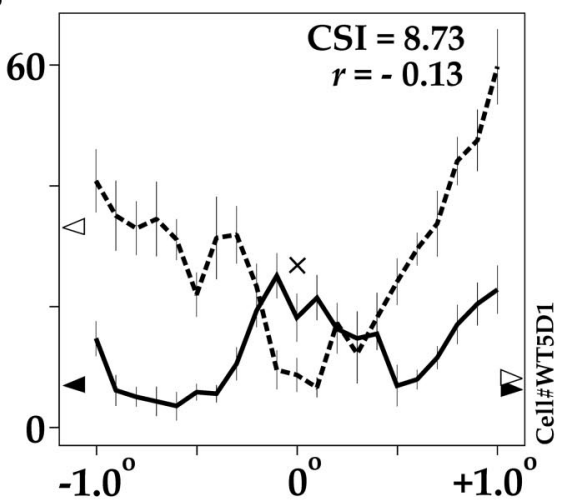

C

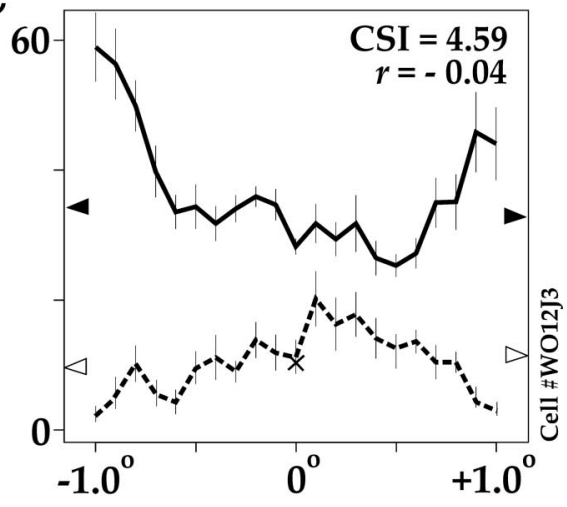

$\mathrm{D}$

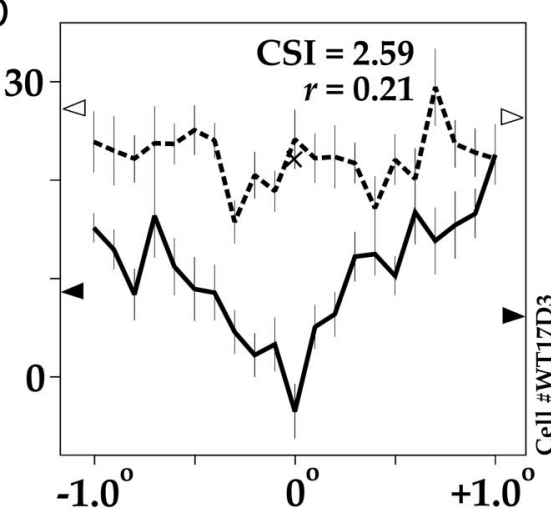

$E$

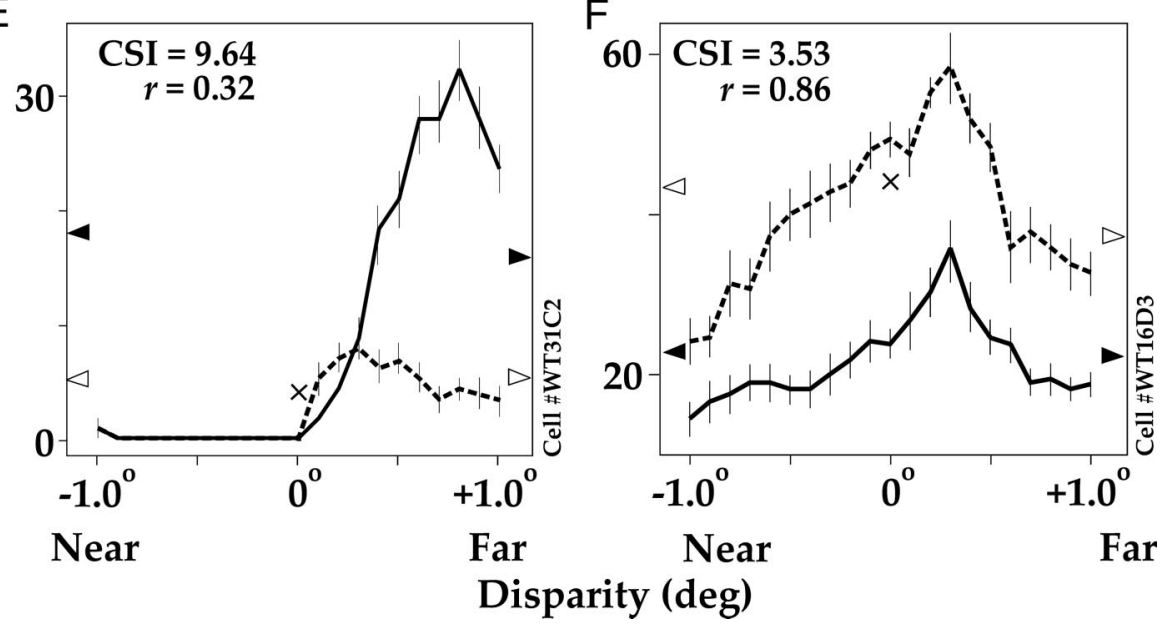

FIG. 2. Disparity tuning of exemplar V4 cells as measured by bars vs. dynamic RDS (dRDS). $A-F$ : responses of individual exemplar cells. In each case, dotted and solid lines denote the cell's disparity tuning profile to dRDS and to bars, respectively. Error bars denote \pm SE. The response modulation across disparities was statistically significant for each of the 6 cells (1-way ANOVAs, $P<$ $0.05)$. The disparity tuning profiles to bars, and dRDS differed significantly from each other for the cells in $B-F$ (2-way ANOVAs, disparity $\times$ stimulus type, $P<0.05$ for disparity, stimulus type, and interaction factors in all 5 cases), but not for the cell shown in $A(P<0.05$ for disparity factor; $P>0.05$ for stimulus type and interaction factors). Responses to bars presented monocularly to the left or the right eye alone are denoted by the solid arrowheads on the left and the right $y$-axes, respectively. Similarly, the open arrowheads on the left and the right $y$-axes denote the cell's responses to dynamic random dot stimuli presented monocularly to left and right eye. $X$ denotes the cell's response to the binocularly uncorrelated dRDS. cells (61\%; Fig. 3, black bars), the disparity tuning was statistically significant $(P<0.05$ for the disparity factor), but the disparity tuning differed significantly between the two sets of stimuli $(P<0.05$ for the interaction factor), and this difference was not attributable to multiplicative scaling (multiplicative scaling test, $P>0.05)$, indicating that these cells conveyed disparity information in a cue-dependent fashion. These cells were classified as cue-dependent. The average CSI value for these 72 cells was 3.7 (data not shown). Nine cells ( $8 \%$; white and hatched bars) were classified as disparity cue insensitive. For seven of these nine cells (6\%; white bars), the disparity tuning was statistically significant, but the two tuning curves were indistinguishable between the two sets of stimuli $(P>0.05$ for the interaction factor). For the other two cells (hatched bars), the two tuning curves were statistically distin- guishable ( $P<0.05$ for the interaction factor), but were multiplicatively scaled versions of each other (multiplicative scaling test, $P<0.05$ ). The remaining 38 cells (31\%; gray bars) were not significantly disparity tuned.

As a further test for scaling effects, we rescaled the two tuning curves for each cell to have identical means and repeated the above analysis. The results (data not shown) were qualitatively similar to those shown in Fig. 3. The average CSI value across all cells was 2.52 . A total of 71 cells $(60 \%)$ was classified as cue sensitive, and the mean CSI value for these cells was 3.31 . Nine cells $(8 \%)$ were classified as cue insensitive, including three cells that were multiplicatively scaled. The remaining 39 cells (33\%) were classified as not significantly tuned for disparity. Thus cue sensitivity of the cells was not attributable to differences in the average responsiveness of 


\begin{tabular}{|c|c|c|c|c|}
\hline & \multicolumn{2}{|c|}{ ANOVA } & \multirow{2}{*}{$\begin{array}{r}\text { Multiplicative } \\
\text { Scaling Test }\end{array}$} \\
\hline & & Disparity & Interaction & \\
\hline Disparity cue sensitive & & $p<0.05$ & $p<0.05$ & $p>0.05$ \\
\hline \multirow[b]{2}{*}{ Disparity cue insensitive } & & $p<0.05$ & $p>0.05$ & $p<0.05$ \\
\hline & vr & $p<0.05$ & $p<0.05$ & $p<0.05$ \\
\hline No disparity selectivity & $\square$ & $p>0.05$ & & \\
\hline
\end{tabular}

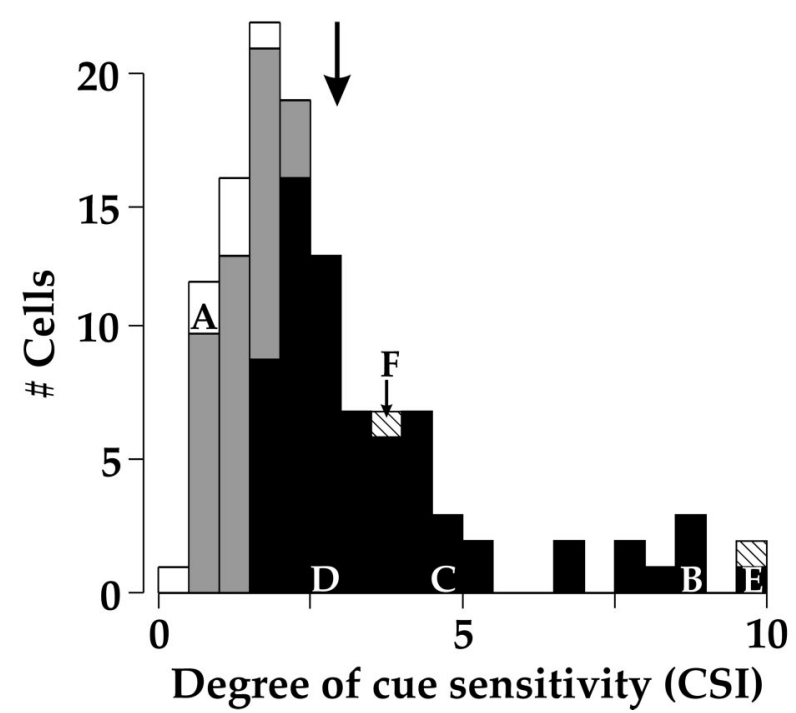

FIG. 3. Disparity cue sensitivity of V4 cells. Degree to which the disparity tuning of a given cell depended on the mapping stimulus (bars vs. dRDS) was measured using the cue sensitivity index (CSI; see METHODS). Distribution of the CSI values is shown here in histogram form. The large arrow denotes the mean CSI for all 119 cells. Cells were further classified as cue sensitive, cue invariant, or as not selective for disparity using a 2-way ANOVA (disparity $X$ stimulus type) and the multiplicative scaling test as shown. Note that, although 8 different categories are possible using the 3 tests at 2 significance levels $(P<$ 0.05 and $P>0.05$ ), the cells are classified using only the 4 categories directly relevant to assessing cue sensitivity. In this figure and the next, the exemplar cells shown in Fig. 2 are denoted by corresponding letters.

cells to bars versus dRDS. To determine whether and to what extent the cue dependency of disparity tuning was attributable to the disparity-dependent differences in the monocular positions of bar stimuli within the CRF (or monocular "artifacts"; see Cumming and DeAngelis 2000), we compared the disparity tuning curves across the four jitter positions using a three-way ANOVA (jitter $\times$ disparity $\times$ stimulus type; data not shown). RDS stimuli minimize this "artifact," since the disparity information in these stimuli is dispersed throughout the stimulus and not confined to the edges, as in the case of the bar stimuli. The jitter factor for bar stimuli was statistically significant $(P<0.05)$ for only a small minority of V4 cells $(6 / 119,5 \%)$ and 4 of the 72 cue-dependent cells $(6 \%)$. For the dRDS stimuli, by comparison, the jitter factor was statistically significant for 9 cells $(8 \%)$ overall and for $6(8 \%)$ of the 72 cue-dependent cells. Thus the cue dependency of disparity tuning is unlikely to have arisen from a widespread sensitivity to the positioning of the stimulus within the CRF.

Another plausible scenario is that, for some cells, the bar stimuli may have stimulated the nonclassical surround with the nonoptimal color. This scenario was possible for 12 cells (10\%), after taking all the relevant factors into account, including the color, length, width, and orientation of the preferred bar, optimal color of the surround, CRF size, stimulus jitter, stimulus disparity, and size of the fixation window. However, the CSI values of these 12 cells were indistinguishable from those of the other cells (Wilcoxon rank-sum test, $P>0.05$ ), indicating that this scenario was unlikely to have played a substantial role in the cue dependency of disparity tuning. The cross-talk between the two monocular views (see METHODS) was unlikely to have been an important factor either, since the magnitude of the cross-talk was generally low and did not affect the CSI values [1-way ANOVA (unbalanced replicates design), $P>0.05]$.

To compare the overall effectiveness of bar versus dRDS stimuli, Fig. $4 A$ plots the peak bar response versus the peak dRDS response, and Fig. $4 B$ plots the mean bar response versus the mean dRDS response. By either measure the two stimulus classes were comparably effective for the population as a whole, but there was only a modest, albeit statistically significant, correlation in the bar versus dRDS responses (correlation coefficient $r=0.38$ for peak responses, 0.35 for mean responses; $P<0.05$ in both cases). Using robust regression, which minimizes the influence of the outliers (see METHODS), the corresponding $r$ values were 0.54 for peak responses and 0.50 for mean responses ( $P>0.05$ in both cases). As is evident from several of the exemplar cells in Fig. 2, the preferred disparities for bars versus dRDS differed for many individual V4 cells. The mean absolute difference in peak disparity value for bars versus $\mathrm{dRDS}$ was $0.74^{\circ}$ (range, $0-2^{\circ}$; 1 st quartile, $0.27^{\circ}$; median, $0.57^{\circ}$; 3 rd quartile, $1.16^{\circ}$ ). A scatterplot of preferred disparities for bar and dRDS disks reveals no significant correlation (Fig. 4C).

Importantly, the three main subclasses of cells (denoted by different symbols in Fig. 4) were statistically indistinguishable from each other in terms of their mean responses, peak responses, or preferred disparities [1-way ANOVA (unbalanced replicates design), $P>0.05$ in all cases; data not shown], indicating that the cue sensitivity, or lack thereof, is unlikely to have arisen from distinct subpopulations of V4 cells.

\section{I S C U S S I O N}

V4 neurons are known to be selective for disparity using bar stimuli (Hinkle and Connor 2001; Watanabe et al. 2002). Disparity selectivity using random dot stimuli has not to our knowledge been tested, nor disparity tuning for bar versus random dot stimuli compared, in V4. Some previous studies have compared the responses of neurons to solid figure versus random dot stimuli in areas V1, V2, V3-V3A (Poggio 1990, 1995; Poggio et al. 1985; also see Gonzalez and Perez 1998; Prince et al. 2002), and area CIP (Taira et al. 2000). While these studies reported many notable differences in the magnitude and the response range of individual neurons to the two types of stimuli, they nonetheless found that the overall tuning profiles were qualitatively similar for the two types of stimuli. Hence, our finding of pronounced stimulus dependence in the disparity domain constitutes a novel finding for V4 in particular and for visual cortex in general. Moreover, the nature and magnitude of the cue dependence reported here differs markedly from that previously reported for motion processing in MT (Olavarria et al. 1992).

It remains to be seen whether and to what extent neurons in other visual areas code disparity in a cue-dependent fashion. 
As noted above, previous studies have found cue-invariant disparity tuning in areas V1, V2, V3-V3A, and CIP. The precise reasons for the discrepancy between these results and ours are difficult to ascertain but may be related to the stimuli and the analytical methods used or genuine differences among areas. It is also possible that disparity selectivity in other areas

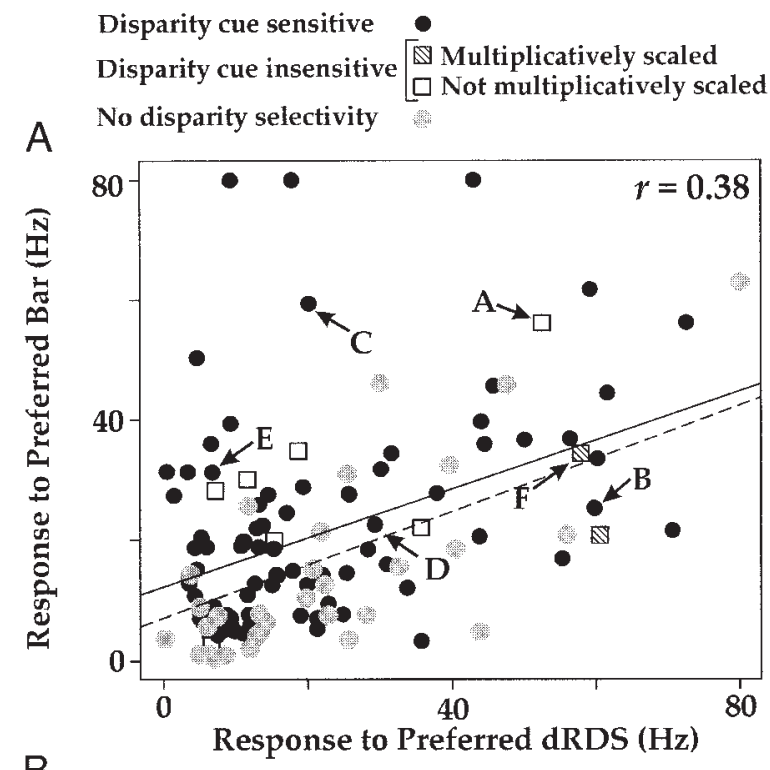

$B$

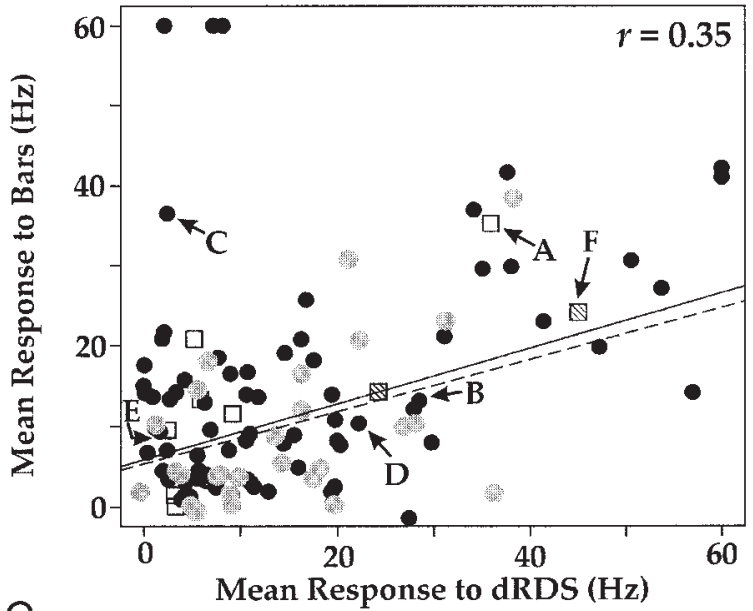

C

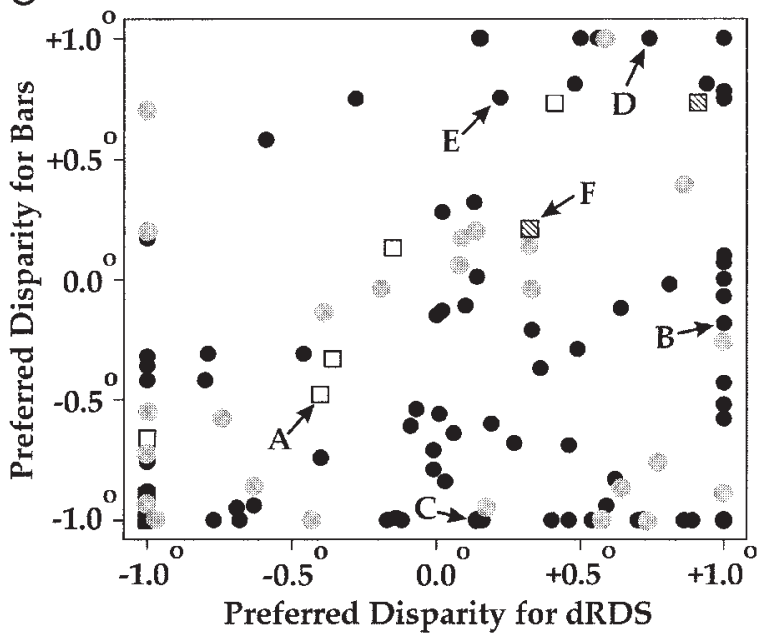

besides V4 will turn out to be cue-dependent when tested with other stimuli (e.g., lines or gratings). By the same token, it is possible that cue dependence of disparity coding is more common in V4 than our results suggest, since at least some of the cells classified as cue invariant or as not selective for disparity in the present study may indeed have been cuedependent for other stimuli. Even if disparity selectivity in V4 turns out to be cue invariant for all other stimuli, our results represent the minimum estimate of cue dependence, and the maximum estimate of cue invariance, of disparity coding in V4. Moreover, the fact remains that the two most common stimulus types used for determining disparity selectivity, i.e., bars and dRDS, often yield widely different disparity tuning curves in area V4.

In interpreting these results, it is important to recognize that the nature and spatial distribution of disparity cues differed markedly for the bars and dRDS used in this study (also see Poggio 1990, 1995). For the bar stimuli, disparity cues were restricted to the perimeter of a rectangular region lying within the CRF (but jittered in exact location from trial to trial). In contrast, the dRDS disparity cues included one disparity value for the random dots throughout the disk-shaped center, a different (albeit constant) disparity value for the annular surround, and a disparity discontinuity along their common boundary. Also, dRDS stimuli by their nature contain numerous false matches involving various dot combinations. Finally, many cells in V2 respond to disparity discontinuities, not just to uniform disparities within the CRF (von der Heydt et al. 2000). Hence, the signals reaching V4 from disparity-sensitive cells in V1 and V2 are likely to be very different for bar versus dRDS disk stimuli.

These considerations suggest several mechanistic factors that might contribute to differences in disparity tuning for bars versus dRDS. If, for example, the disparity tuning within the CRF of a V4 cell is spatially nonhomogeneous, bar stimuli might predominantly activate a subregion of one type (e.g., a tuned excitatory strip), whereas the dRDS disk might activate a combination of subregions having different disparity preferences. Differential activation of subregions might persist even with the trial-to-trial jittering of stimulus positions (see METHODS) if the subregions encompass a substantial fraction of the CRF. If V4 cells are sensitive to depth discontinuities, as occurs in some V2 neurons, the tuning for mean disparity within the CRF might be modulated by the depth discontinuities of the disk/annulus border. Another possible factor is an influence of tuning for relative disparity, rather than strictly absolute disparity, as has been reported for some V2 cells (Thomas et al. 2002). Relative disparity tuning might be expected to sharpen the tuning profiles for dRDS (which include a reference disparity in the annular region) compared with bar stimuli (where the fixation point is the only reference

FIG. 4. Comparisons of responses and preferred disparities of V4 cells for bars vs. dRDS disks. $A$ : peak responses for bar vs. dRDS disks. $B$ : mean responses above background for bars vs. dRDS disks. Outliers $>80 \mathrm{~Hz}$ were plotted at $80 \mathrm{~Hz}$ in $A$, and outliers $>60 \mathrm{~Hz}$ were plotted at $60 \mathrm{~Hz}$ in $B$. Solid lines denote the best-fitting regression lines, calculated with outliers at their actual values. Dashed lines denote robust regression lines. $C$ : preferred disparities for bars vs. dRDS disks. To reduce the uncertainty in the estimated preferred disparity arising from to trial-to-trial fluctuations in responsiveness and from multiple peaks in some of the response profiles, the preferred disparities were calculated from tuning curves smoothed using a Gaussian filter $\left(\sigma=0.1^{\circ}\right)$. See METHODS for details. 
disparity); however, this is not the primary difference between the tuning profiles encountered in our study (see Fig. 2).

These issues can be further investigated by tests that were not included in our original paradigm, because it was designed to explore a different set of issues relating to three-dimensional shape processing (unpublished data). For example, it would be of interest to quantitatively compare tuning profiles using luminance versus random dot stimuli that represent similar geometric shapes (e.g., bars for both or disks for both; see Poggio 1990, 1995; Prince et al. 2002; Taira et al. 2000).

Irrespective of the mechanistic issues, it is of interest to know what role, if any, cue-dependent disparity tuning plays in three-dimensional form perception. Conceivably, the stimulus dependence reported here might be epiphenomenal, if the visual system could somehow make use of selectivities for shape, color, and other attributes while ignoring the disparityrelated tuning of cells in V4. However, it seems implausible that this explanation would apply generically to all cue-dependent cells in V4. Alternatively, disparity tuned cells in V4 may contribute to stereoscopic depth perception by a process that entails a more complex decoding strategy for inferring depth and shape. This might involve the establishment of cue-independent tuning in individual neurons at higher stages (e.g., in inferotemporal cortex) or by cue independence that is manifest at a population level rather than in individual tuning profiles.

\section{A C K N O W L E D G M EN T S}

We thank Dr. Greg DeAngelis for advice and help throughout the project, Drs. Ed Connor and David Hinkle and D. Hanlon for help with UNIX hardware and software, A. Anzai for comments on the manuscript, and S. Danker for assistance in manuscript preparation.

Present address of J. Hegdé: Vision Center Laboratory, The Salk Institute for Biological Studies, 10010 N. Torrey Pines Rd., La Jolla, CA 92037.

\section{G R A N T S}

This work was supported by National Eye Institute Grant EY-02091.

\section{REFEREN CES}

Albright TD. Form-cue invariant motion processing in primate visual cortex. Science 255: 1141-1143, 1992.

Cumming BG and DeAngelis GC. The physiology of stereopsis. Аnпи Rev Neurosci 24: 203-238, 2000.

Cumming BG and Parker AJ. Responses of primary visual cortical neurons to binocular disparity without depth perception. Nature 389: 280-283, 1997.

Dean AF. The variability of discharge of simple cells in the cat striate cortex. Exp Brain Res 44: 437-440, 1981.

Dobkins KR and Albright TD. What happens if it changes color when it moves?: psychophysical experiments on the nature of chromatic input to motion detectors. Vision Res 33: 1019-1036, 1993.

Edgington ES. Randomization Tests. New York: Dekker, 1995.
Gattas R, Souza APB, and Gross CG. Visuotopic organization and extent of V3 and V4 of the macaque. J Neurosci 8: 1831-1844, 1988.

Gonzalez F and Perez R. Neural mechanisms underlying stereoscopic vision. Prog Neurobiol 55: 191-224, 1998.

Hegdé $\mathbf{J}$ and Van Essen DC. Selectivity for complex shapes in primate visual area V2. J Neurosci 20: RC61-RC66, 2000.

Hegdé $\mathbf{J}$ and Van Essen DC. Strategies of shape representation in macaque visual area V2. Vis Neuroci 20: 313-328, 2003.

Hinkle DA and Connor CE. Disparity tuning in macaque area V4. Neuroreport 12: 365-369, 2001.

Manly BFJ. Randomization and Monte-Carlo Methods in Biology. New York: Chapman and Hall, 1991.

Masson GS, Busettini C, and Miles FA. Vergence eye movements in response to binocular disparity without depth perception. Nature 389: 283-286, 1997.

McAdams CJ and Maunsell JH. Effects of attention on orientation-tuning functions of single neurons in macaque cortical area V4. J Neurosci 19: 431-441, 1999.

Olavarria JF, DeYoe EA, Knierim JJ, Fox JM, and Van Essen DC. Neuronal responses to visual texture patterns in middle temporal area of the macaque monkey. J Neurophysiol 68: 164-180, 1992.

Parker AJ and Cumming BG. Cortical mechanisms of binocular stereoscopic vision. Prog Brain Res 134: 205-216, 2001.

Poggio GF. Cortical neural mechanisms of stereopsis studied with dynamic random-dot stereograms. Cold Spring Harbor Symp Quant Biol 55: 749758, 1990.

Poggio GF. Mechanisms of stereopsis in monkey visual cortex. Cereb Cortex 5: 193-204, 1995.

Poggio GF, Motter BC, Squatrito S, and Trotter Y. Responses of neurons in visual cortex (V1 and V2) of the alert macaque to dynamic random-dot stereograms. Vision Res 25: 397-406, 1985.

Prince SJ, Cumming BG, and Parker AJ. Range and mechanism of encoding of horizontal disparity in macaque V1. J Neurophysiol 87: 209221, 2002.

Rousseeuw PJ. Least median of squares regression. J Am Stat Assoc 79: 871-881, 1984.

Sáry G, Vogels R, and Orban GA. Cue-invariant shape selectivity of macaque inferior temporal neurons. Science 260: 995-997, 1993.

Stoner GR and Albright TD. Motion coherency rules are form-cue invariant. Vision Res 32: 465-475, 1992.

Stoner GR and Albright TD. Image segmentation cues in motion processing: implications for modularity in vision. J Cogn Neurosci 5: 129-149, 1993.

Taira M, Tsutsui KI, Jiang M, Yara K, and Sakata H. Parietal neurons represent surface orientation from the gradient of binocular disparity. J Neurophysiol 83: 3140-3146, 2000.

Tanaka H, Uka T, Yoshiyama K, Kato M, and Fujita I. Processing of shape defined by disparity in monkey inferior temporal cortex. J Neurophysiol 85: 735-744, 2001.

Thomas OM, Cumming BG, and Parker AJ. A specialization for relative disparity in V2. Nat Neurosci 5: 472-478, 2002.

Van Essen DC and Zeki S. The topographic organization of rhesus monkey prestriate cortex. J Physiol 277: 193-226, 1978.

von der Heydt R, Zhou H, and Friedman HS. Representation of stereoscopic edges in monkey visual cortex. Vision Res 40: 1955-1967, 2000.

Watanabe M, Tanaka H, Uka T, and Fujita I. Disparity-selective neurons in area V4 of macaque monkeys. J Neurophysiol 7: 1960-1973, 2002. 